\title{
Does Prenatal Stress Management Enhance Maternal And Fetal Outcomes?
}

\author{
Ghada Mohammad Abu Shosha, PhD
}

Faculty of Nursing, Zarqa University, Jordan

doi: 10.19044/esj.2016.v12n27p129 URL:http://dx.doi.org/10.19044/esj.2016.v12n27p129

\begin{abstract}
Background: Antenatal period is a time of rapid change during which fetal organs are vulnerable to various stressors. Studies have suggested that psychosocial stressors during pregnancy could adversely influence physical and behavioral outcomes of the infant. Aim: This paper aimed to discusses the importance of antenatal stress assessment and management on both mothers' and infants' health status. Method: This review aggregated evidence from various studies that examined the impact of maternal stress management and its outcomes on pregnant women and their infants. Results: Maternal stress is generally associated with unpleasant fetal outcomes. The use of stress reduction techniques was approved to reduce psychological stress in pregnant women. Conclusion: Ongoing assessment of antenatal stress using a standardized process promotes proper stress handling strategy.
\end{abstract}

Keywords: Antenatal period, Stress, Stress management

\section{Introduction}

Antenatal period is a time of rapid changes in which fetal organs are developing and it is a critical stage affected by various influences (Davis \& Sandman, 2010). Experimental studies suggest that the psychosocial stressors during pregnancy can influence physical, behavioral and cognitive outcomes of the offspring (King \& Laplante, 2005).

Many services are provided for women during pregnancy through antenatal clinics to have best maternal and fetal outcomes. According to the World Health Organization (WHO, 2003); $78 \%$ of women in the developing world receive antenatal care from skillful health providers at least for once during pregnancy to ensure high quality antenatal care and to preserve maternal and fetal outcomes.

The antenatal period provides essential opportunities including a number of interventions that may enhance health and well-being for pregnant women and infants (Abouzahr \& Wardlaw, 2003). Regular follow up by 
health care providers contributes in the following manners; treatment of hypertension to prevent eclampsia, tetanus immunization, intermittent preventive treatment for malaria and distribution of insecticide-treated mosquito nets, prevention of mother to child transmission of human immunodeficiency virus, micronutrient supplementation and birth preparedness including information about danger signs during pregnancy and childbirth (WHO, 2003). The antenatal care also provides an opportunity to give information about birth spacing, which is defined as an important factor of providing better infant survival (WHO, 2003). However, stress education is less recognized within these programs.

Moos (2006) reviewed the evolution occurred in prenatal care between the years 1902 to 2005. Moos reinforced early activities recommended to pregnant women including assessment of danger signs, checking the blood pressure and urine, assessing fetal heart tones and providing nutritional counseling, hygiene, exercise, and preparing for infant care. After World War II, the concerns of infant mortality were increased and prenatal care has been introduced as a factor of reducing prematurity and low birth weight. Recently, many women and infants benefit from prenatal care to achieve the secondary prevention, such as; receiving RhoGAM to prevent isoimmunization, maternal antibiotic treatment to prevent congenital syphilis, maternal antiviral drugs to diminish the risk of vertical transmission of human immunodeficiency virus, and maternal influenza vaccine to prevent pregnancy complications (Moos, 2006).

On the other hand, the United States Public Health Service (PHS, 1989) has assigned a panel of experts to determine aspects of prenatal care that should be preserved, and what was needed and should be instituted, and components that were not useful or helpful to be discarded. The panel has recommended three main components of antenatal care; early and continuous risk assessment, health promotion, and medical and psychosocial interventions and follow up. The panel asserted that risk assessment during antenatal care must include; (1) medical risk factors such as genetic factors and reproductive history; and (2) psychosocial risk factors including socioeconomic status, psychological factors, and adverse health habits. However, the panel on the content of antenatal care placed attention on assessment of maternal stress and emotional problems during pregnancy as these factors may play a role in the result of delivery and birth process as well as fetal outcomes (PHS, 1989).

Yet, several organizations and institutions developed holistic guidelines of the content of antenatal care. Unfortunately, none of these focused on assessment of maternal stress and stress management strategies during pregnancy. According to WHO (2003) guidelines, antenatal care visits should include at least the measurement of blood pressure, testing of 
urine for bacteriuria and proteinuria, and blood tests to detect syphilis and severe anemia. Similarly, the National Institute for Health and Clinical Excellence (NICE, 2008) asserted that healthcare providers should remain vigilant to the risk factors, signs and symptoms of conditions might affect mother child health. Therefore, the NICE (2008) established guidelines including; management of common pregnancy symptoms, clinical examination of pregnant women, screening for hematological conditions, screening for fetal abnormalities, screening for infections disorders, screening for other clinical features and assessment of fetal growth and wellbeing.

Despite the emphasis on managing pregnant women physiologically, most of these guidelines excluded the importance of psychological assessment and emotional support during pregnancy.

\section{The effect of prenatal stress on pregnancy outcomes}

Many studies have examined the effect of prenatal stress on pregnancy outcomes (Hobel \& Culhane, 2003; Diego et al., 2006; Grizenko et al., 2008; Davis \& Sandman; 2010; \& Leung et al., 2010).

Hobel \& Culhane (2003) reviewed the epidemiological and clinical research evidence related to maternal stressors including perceived stress, life event stress, pregnancy-related anxiety and work stress, and nutritional stress to adverse outcomes of pregnancy. The authors have noted a progression of empirical evidence based on different ethnics, socioeconomic and cultural backgrounds, and supporting the premise that mothers experienced a high level of psychosocial stress during pregnancy are at risk of deteriorating preterm birth even after adjusting the effects of other risk factors. According to the reviewed studies, stress could results in elevations of pituitary peptides hormones, corticotropin-releasing hormone $(\mathrm{CRH})$ and adrenocorticotropin hormone (ACTH) leading to elevations in cortisol level in blood and glucose as well. Elevation in $\mathrm{CRH}$ has shown a positive association with stress scores in patients with preterm delivery compared with a full term delivery, also excessive cortisol level showed an association with decreased fetal growth (Hobel \& Culhane, 2003).

Similarly, Diego et al. (2006) conducted a cross-sectional study to examine the effects of maternal psychological distress during midgestation on fetal development by assessing the relations between fetal growth measures and maternal stress, depression, and anxiety during midgestation. Correlation analysis revealed that maternal psychological stress, depression, and anxiety and biochemical variables including cortisol and norepinephrine were correlated negatively with fetal biometrical measurements and estimated fetal weight. In conclusion, women exhibiting psychological 
distress with elevated cortisol levels during midgestation have lower fetal weight.

Davis \& Sandman (2010) examined the consequences of prenatal maternal stress on infant mental and motor development in 125 full term infants at 3, 6 and12 months of age. Maternal cortisol and psychological status were assessed five times during pregnancy. Exposure to early elevation of cortisol in gestation was associated with slower development rate over the first postnatal year and lowered scores on the mental development index of the Bayley Scales of Infant Development (BSID) at 12 months. Also, elevated levels of early maternal anxiety were independently associated with lower scores on the BSID at 12 months. On the other hand, Grizenko et al. (2008) examined whether there is an association between the severity of maternal stress and the severity of symptoms of attention-deficit hyperactivity disorder (ADHD). The authors found that children with ADHD whose mothers were exposed to moderate to severe stress during pregnancy tend to develop more serious symptoms than children with ADHD whose mothers were not exposed to prenatal stress.

Finally, Leung et al. (2010) examined the relation between perceived maternal stress during pregnancy and stability of infant stress reactivity across the first year of postnatal life. The study found that maternal stress during pregnancy predicted infant cortisol reactivity at 2 days and 10 months after birth as well as behavioral reactivity at 10 months.

\section{Discussion}

Maternal psychological stress has negatively impacted on fetal health outcomes. The majority of studies reported that a high level of maternal stress is associated with negative fetal outcomes. These outcomes included hormonal disturbances in both mother and fetus (i.e. higher cortisol level) that impair fetal development. These findings should encourage health care providers to assess and reduce maternal stress thoroughly. Thus, antenatal services should include assessment of maternal stress and emotional status as well as application of stress reduction techniques. Stress reduction techniques such as aerobic exercise, could reduce psychological stress in pregnant women (Lox \& Treasure, 2000). Jallo et al. (2008) introduced a12 week study to determine whether a relaxation guided imagery (RGI) intervention could be used as a preventive measure for maternal stress. RGI was found beneficial to women during the second trimester of pregnancy. All participants acknowledged the benefits of the RGI intervention including the following; (1) improved breathing; (2) ability to relax ; (3) ability to control and decrease stress; (4) release of anxiety; (5) additionally, use of RGI throughout the day helped control anger and state of mind; and (6) improved ability to stay sleeping. 
The recommended screening of maternal stress should include an identification of women experiencing stress and a determination of stress sources. Referral to a specialized stress management services such as social support, prenatal education, and stress management techniques can be recommended.

\section{Conclusion}

Evidences support the idea that stress during pregnancy results in drawback effects on both mothers and infants. Assessing pregnant women's stress and applying stress reduction techniques when needed were found beneficial. Therefore, health care professionals should be oriented on the negative consequences associated with maternal stress during pregnancy and its impact on infant' physical and mental development.

\section{References:}

Abouzahr, C. and Wardlaw, T. (2003). Antenatal care in developing countries : promises, achievements and missed opportunities : An analysis of trends, levels and differentials,1990-2001. WHO Library Cataloguing-inPublication Data.

Davis, E., and Sandman, C. (2010). The timing of prenatal exposure to maternal cortisol and psychosocial stress is associated with human infant cognitive development. Child Development, 81(1), 131-148.

Diego, M., Jones, N., Field, T., Hernandez, M., Schanberg, S., and Garcia, A. (2006). Maternal psychological distress, prenatal cortisol, and fetal weight. Psychosomatic Medicine, 68:747-753.

Grizenko, N., Shayan, Y., Polotskaia, A., Ter-Stepanian, M., Joober, R.(2008). Relation of maternal stress during pregnancy to symptom severity and response to treatment in children with ADHD. Journal of Psychiatry \& Neuroscience, 33(1),10-16.

Hobel, C., and Culhane, J. (2003). Life and fitness. Role of psychosocial and nutritional stress on poor pregnancy outcome. The journal of Nutrition. 1709-1717.

Jallo, N., Bourguignon, C., Taylor, G., and Utz, S. (2008). Stress management during pregnancy: Designing and evaluating a mind-body Intervention. Family \& Community Health, 31(3), 190-203.

King, S. \& Laplante, D. (2005). The effects of prenatal maternal stress on children's cognitive development: Project Ice Storm. Stress, 8(1), 35-45.

Lox, C. L.,\& Treasure, D. C. (2000). Changes in feeling states following aquatic exercise during pregnancy. Journal of Applied Social Psychology, 30(3), 518-527.

Leung, E., Tasker, S., Atkinson, L., Vaillancourt, T., Schulkin, J., and Schmidt, L. (2010). Perceived maternal stress during pregnancy and its 
relation to infant stress reactivity at 2 days and 10 months of postnatal life. Clinical Pediatrics, 2010; 49: 158- 165.

Moos, M., (2006). Prenatal Care: Limitations and opportunities, Journal of Obstetric, Gynecologic, \& Neonatal Nursing, 35(2), 278- 285

National Institute for Health and Clinical Excellence(2008). Antenatal care: routine care for the healthy pregnant woman. Available at: http://www.pennine-gp- training.co.uk/Antenatal-Care-2008.doc.

United States Public Health Service, (1989). Caring for our future: The content of prenatal care; a report of the public health service expert on the content of prenatal care. Department of health and human service. Washington, DC.

World Health Organization (2003). Antenatal care in developing countries. Available at: http://www.childinfo.org/files/antenatal_care.pdf. 